\title{
Extraktion der Inzisivi bei Kaninchen
}

\author{
Stefan Gabriel
}

Primäre oder sekundäre Malokklusionen können bei Kaninchen zum übermäßigen Wachstum der Inzisivi führen. Die zu lang gewachsenen und gekrümmten Schneidezähne verlieren ihre Funktion und stellen nur noch ein Hindernis bei der Nahrungsaufnahme dar. Die Extraktion ist die einzig sinnvolle Therapie funktionslos gewordener Inzisivi.

Das gesamte Gebiss der Lagomorpha besteht aus permanent nachwachsenden Zähnen und schärft sich selbst. Physiologischerweise besteht zwischen Abrieb und Nachwachstum ein feines Gleichgewicht, das durch falsche Fütterung und selektives Fressen bei zu reichhaltigem Nahrungsangebot kippt. Neben der primären, angeborenen Malokklusion bei brachyzephalen extremen Zwergen führt vor allem die sekundäre, erworbene Malokklusion zu behandlungsbedürftigen Zahnfehlstellungen. Dabei kommt es durch eine zu hohe Bisslage im Backenzahngebiss sekundär zu einer Veränderung im Schneidezahngebiss: Die Elongation der Molaren führt zu einem über- mäßigen Längenwachstum der Inzisivi. Die elongierten Inzisivi $\mathrm{I}_{1}$ im Oberkiefer entwickeln eine größere Krümmung und dabei entstehen häufig auch die pathognomonischen Querriefen. Im Unterkiefer entwickeln die Inzisivi eine geringere Krümmung und wachsen zunehmend vor die Antagonisten im Oberkiefer, wobei schließlich der physiologische Kontaktpunkt auf der Hinterseite des $\mathrm{I}_{1}$ und damit die Funktion vollständig verloren gehen ( $\triangleright$ Abb. 1). Diese Veränderungen werden heute als Stadien einer chronisch-progressiv verlaufenden Dentalerkrankung betrachtet [7].

\section{Indikationsstellung}

Funktionslos gewordene Inzisivi lassen sich wegen der eingetretenen morphologischen Veränderungen und häufig zusätzlicher parodontaler Schädigung (Rotation und/oder Lockerung in den knöchernen Alveolen, Abb.2) nicht mehr durch Einschleifen ad integrum restituieren und stellen letztlich nur noch ein Hindernis bei der Nahrungsaufnahme dar. Meist werden diese fälschlich als
„Elefantenzähne“ bezeichnet und regelmäßig gekürzt. Keinesfalls darf diese Kürzung mit Zangen oder Clippern durchgeführt werden, weil die Inzisivi zu Längssplitterungen neigen und das $\mathrm{Ab}$ kneifen hoch schmerzhaft ist. Auch regelmäßiges Absägen mit der Diamattrennscheibe ist keine gute Option, weil die Pulpa überlanger Zähne ebenfalls bis weit über das Gingivaniveau elongieren kann. So besteht beim Kürzen dieser Zähne ein hohes Risiko, die Zahnpulpa zu eröffnen und iatrogen eine Pulpitis zu verursachen. Abb. 3 zeigt ein Paar solcher regelmäßig abgesägten Inzisivi mit chronischer offener (iatrogener!) Pulpitis.

\section{Die einzig sinnvolle Therapie für}

funktionslose Inzisivi ist die Beseitigung dieser Kauhindernisse durch Extraktion.

Dadurch kann die Lebensqualität des Patienten weitgehend normalisiert werden $[1,7,8]$. Voraussetzung für die Extraktion der Inzisivi ist jedoch ein normaler oder prognostisch günstiger Befund der Backenzähne.

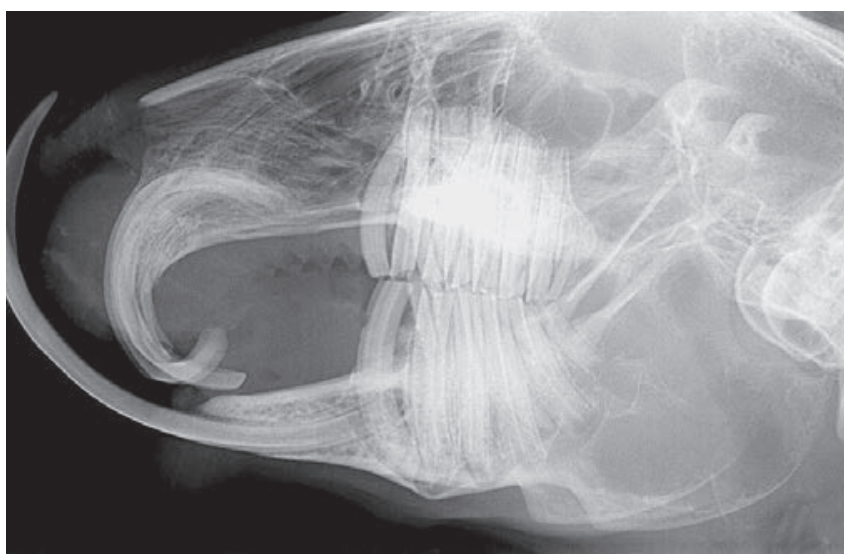

Abb. 1 Totaler Kontaktverlust der Inzisivi und deutliche Elongation der Backenzähne mit retrograden Wurzelveränderungen. Divergierende Konturen von Gaumen und Unterkieferdiastema. Die Inzisivi sind völlig funktionslos und behindern nur die Nahrungsaufnahme. (c S. Gabriel

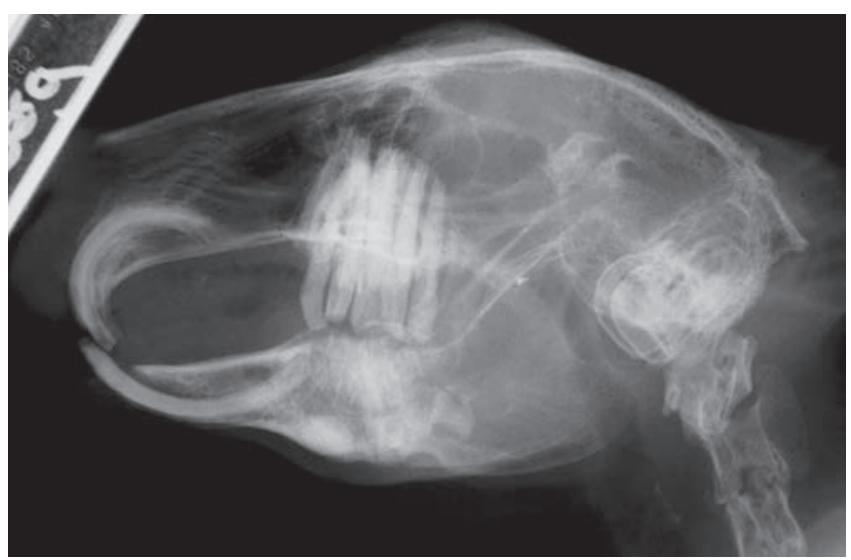

Abb. 2 Malokklusion der Inzisivi 3. Grades mit parodontaler Lockerung der Unterkieferinzisivi, Rotation und Intrusion in den großen Unterkieferabszess. (C) M. Lampe 


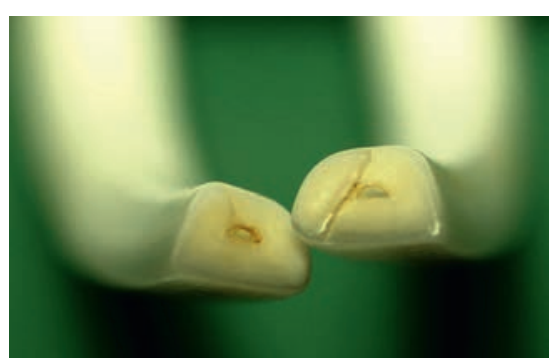

Abb. 3 Extrahierte elongierte Unterkieferinzisivi. Der Haustierarzt hatte die vermeintlichen „Elefantenzähne“ im Abstand von 6 Wochen mit der Trennscheibe gekappt und unbemerkt eine offene Pulpitis unterhalten. (C) S. Gabriel

Vereiterte oder im Kiefer gelockerte Inzisivi stellen eine absolute Indikation zur Extraktion dar. Entfernt man diese schmerzhaften Zähne nicht, kommt es zu Frakturen, Ausbrechen oder einer Intrusion in den Kieferknochen ( $\triangleright$ Abb.4).

\section{Röntgendiagnostik}

Zur Beurteilung der Länge und Form der zu extrahierenden Inzisivi muss vor dem Eingriff mindestens eine laterale Schädelaufnahme angefertigt werden [5]. Diese Aufnahme dient zur:

- Auswahl der passenden Werkzeuge

- Kontrolle der jeweiligen Arbeitstiefe $($ Abb. 5)

Anhand von Referenzlinien [2,3] lässt sich die Bisslage der Backenzähne kontrollieren. Eine postoperative Röntgenkontrolle dokumentiert die vollständige
Entfernung der Zahnhartsubstanzen und die Integrität der Kieferknochen [1, 4, 8].

\section{Narkose und Analgesie}

Eine ausreichend tiefe Allgemeinnarkose zur entspannten Lagerung kann durch eine Isofluran-Inhalationsnarkose oder eine Injektionsnarkose erfolgen.

Beispiele möglicher Injektionsnarkosen sind:

- die total antagonisierbare Triple-Narkose: Medetomidin 0,2 mg/kg + Midazolam 1,0 mg/kg + Fentanyl 0,025 mg/ kg i.m. [10]

- ihre Modifikation ohne Fentanyl: Medetomidin 0,1-0,25 mg/kg + Midazolam 0,5-1,0mg/kg + Butorphanol 0,4$0,6 \mathrm{mg} / \mathrm{kg}$ i.m.

Dabei ist zu beachten, dass Isofluran allein nicht analgetisch wirkt und nach Antagonisierung des Opiatanteils der Triple-Narkose auch die Analgesie endet [9]. Eine sorgfältige perioperative Analgesie z.B. mit präemptiver Gabe von Butorphanol und postoperativer Fortsetzung mit Metamizol (20-50 mg/kg/4h) und einem der beiden NSAIDS hat sich bewährt [6]:

- Meloxicam: 0,3-1 mg/kg/24h

- Carprofen: 4-5 mg/kg/24h

Die lokale Betäubung als Leitungs- oder Infiltrationsanästhesie bietet sich zusätzlich an. Vorzugsweise sollte sie mit einer speziellen intraligamentalen Injektionsspritze appliziert werden. Diese zahnärztliche Repetierspritze setzt bei jeder Betätigung ein definiertes Volumen von z.B. 0,1 ml Lokalanästhetikum unter Druck ins Gewebe ab, ohne dass irrtümlich eine größere Menge intravasal abfließen kann. Mit der zugehörigen extrem dünnen Zahnarztkanüle kann man die Kiefernerven (Unterkiefer: N. mentalis, Oberkiefer: N. infraorbitalis) durch ein periostnahes Depot blocken. Auch eine intraligamentale Lokalinjektion in die Parodontalspalten der Inzisivi ist mit einer langen Zahnarztkanüle möglich ( Video '머).

Eine intraoperative Verabreichung von Sauerstoff wird bei Kaninchennarkosen stets empfohlen. Da ein Trachealtubus bei der Zahnextraktion hinderlich ist, kann eine nasopharyngeale Intubation mit einer dünnen Ernährungssonde erfolgen ( $\triangleright$ s. Kasten). Über diese Sonde kann leicht Sauerstoff, aber auch zur Narkosevertiefung zusätzlich Isofluran insuffliert werden.

\section{Instrumentarium}

Während in der Literatur [1,4] meist noch auf selbstgefertigte Luxationswerkzeuge oder umgearbeitete Zahnarztspatel zurückgegriffen wird, stehen heutzutage spezielle Nagerzahnluxatoren diverser Hersteller zur Verfügung (Bezug z.B. über Albrecht, Henry Schein,

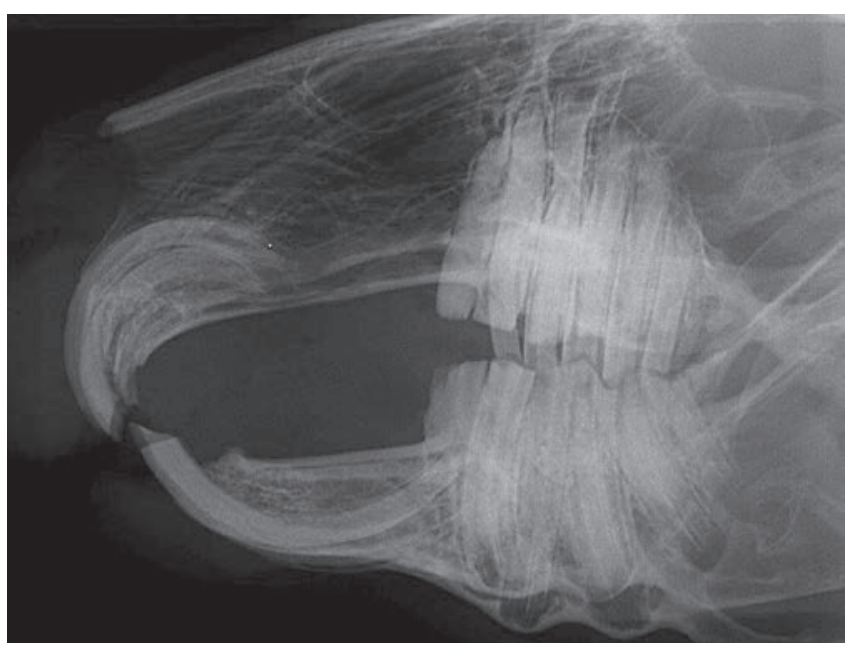

Abb. 4 Rotation der Inzisivi im Kiefer. Beginnende Perforation der Compacta des Gaumens und Wurzelverlagerung des Unterkieferinzisivus. (C) S. Gabriel

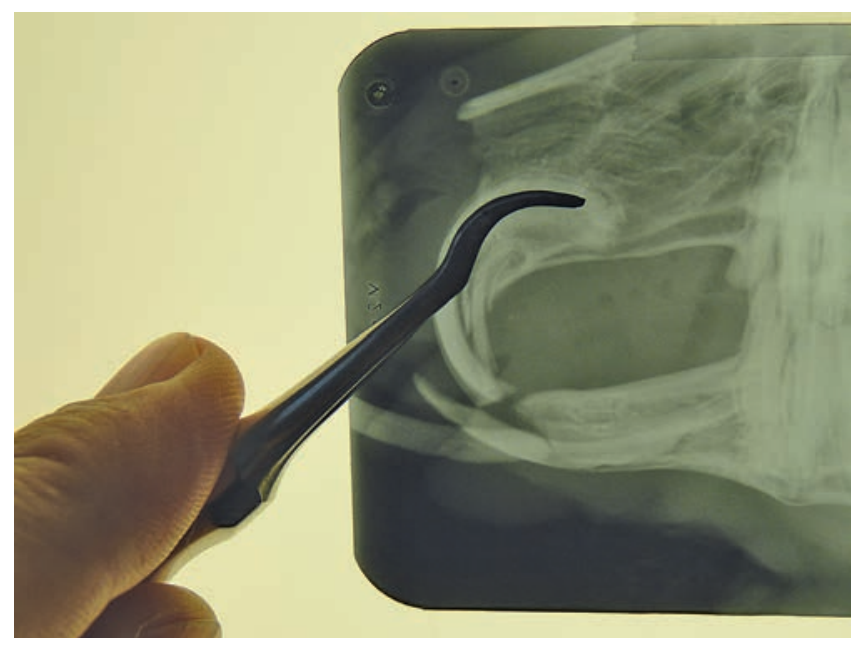

Abb. 5 Auswahl des passenden Luxationsinstruments am Röntgenbild und Abmessung der erforderlichen Arbeitstiefe. (c) S. Gabriel 


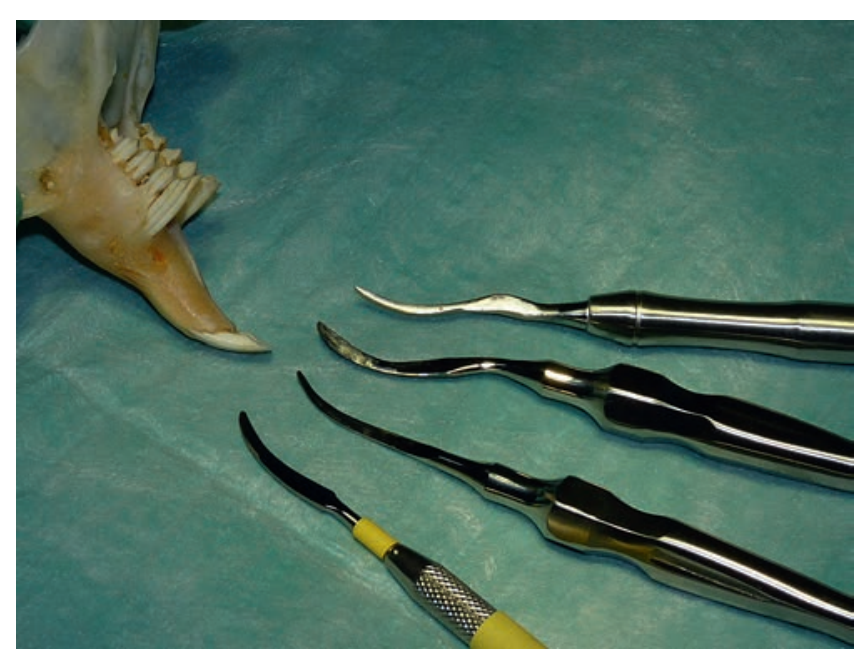

Abb. 6 Verschiedene Luxatoren. Von unten: nach Crossley, nach Remeeus, nach Fahrenkrug (teilweise modifiziert), Eigenbau nach Gabriel. (c) S. Gabriel

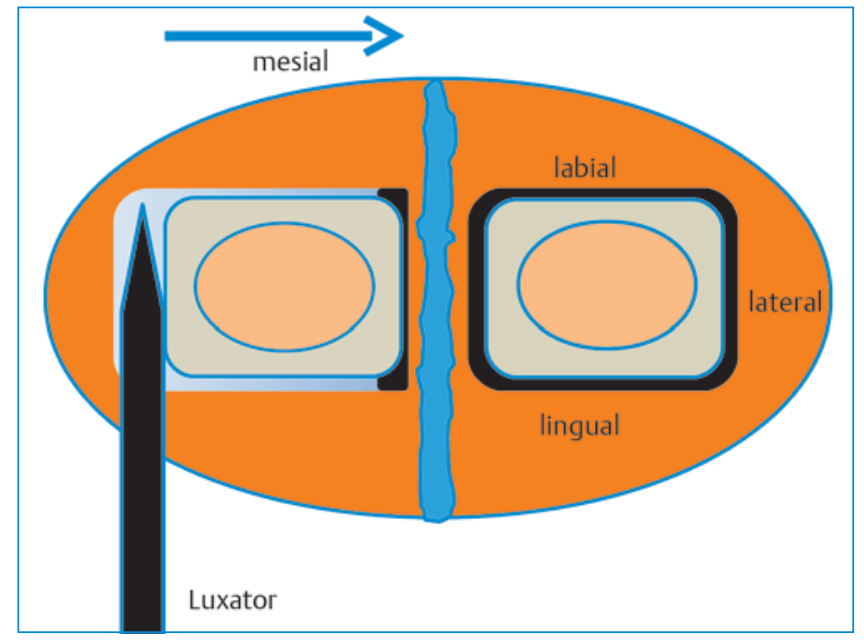

Abb. 7 Schematischer Querschnitt durch den Unterkiefer mit der Symphyse in der Mitte. Der Luxator wird lateral in den Parodontalspalt eingeführt und luxiert den Inzisivus nach mesial, wobei die parodontalen Fasern reißen. Als Nächstes erfolgt die Gegenbewegung nach lateral durch die mesiale Einführung des Luxators. (C) S. Gabriel
WDT/IM3). Im Unterschied zu den konventionellen Hebeln (Elevatoren) für die Zahnextraktion bei Hund und Katze handelt es sich hierbei um kleine sichelförmige Messer (Periotome), die nicht zum Hebeln, sondern zum Schneiden (Luxieren) in der Alveole konzipiert sind. Daher sollten die stumpf gelieferten Luxatoren vor jedem Gebrauch an ihrer Spitze angeschliffen werden. Ein Satz dieser Nagerzahnluxatoren besteht aus 2-3 doppelendigen Instrumenten mit verschiedenen Krümmungen ( $\bullet$ Abb. 6).

Da die parodontalen Fasern den Zahn nicht fest mit der knöchernen Alveole verbinden, ist das Ziel der Luxation, unter
Aufweitung des Parodontalspalts bis zum Wurzelbereich herunter zu dringen und dort mit der scharfen Spitze des Instruments den Zahn loszuschneiden. Er lässt sich dann einfach aus seiner Wurzelscheide herausziehen $[7,8]$.

\section{OP-Vorgehensweise}

Der anästhesierte Kaninchenpatient wird in Seitenlage auf einer körperwarmen Unterlage vorbereitet und alle Instrumente werden bereitgelegt. Optimal liegt das seitliche Röntgenbild zur Kontrolle der Arbeitstiefe in der Nähe auf einem Bildbetrachter bereit.

\section{Nasopharyngeale Intubation}

Eine passende Ernährungssonde (Außendurchmesser 1-3 mm) wird zunächst für den Patienten abgemessen. Dazu misst man die Distanz vom Nasenloch bis zum temporalen Augenwinkel ab und markiert diese auf der Sonde mit einem Filzstift. Unter Verwendung von etwas Gleitgel oder Lidocain-Gel schiebt man nun die Sonde beim anästhesierten Patienten durch den ventralen Nasengang bis zur Markierung in den Nasopharyx vor. Durch vorsichtiges Einpustenwerden Gel und Schleimreste von der Sondenspitze beseitigt. Die richtige Lage der Sonde wird entweder mithilfe eines Kapnometers (falls vorhanden) oder durch Beobachtung des Beschlagens durch die Atemluft im Tubus überprüft. Wenn man eine röntgenfähige Sonde verwendet, lässt sich im Zweifelsfall der korrekte Sitz am einfachsten mit einer Röntgenaufnahme kontrollieren. Mit einem Klebestreifen wird die Sonde an der Nase fixiert und vor Verrutschen bei den Umlagerungen gesichert. Mit einem Adapter kann nun sowohl Sauerstoff (kontinuierlich) als auch Isofluran (nach Bedarf) in geringem Fluss insuffliert werden.

Cave: eine irrtümliche ösophageale Fehlintubation muss sicher verhindert werden!!!

Eine nasopharyngeale Sonde, über die Sauerstoff und bei Bedarf auch Isofluran zur temporären Narkosevertiefung gegeben werden kann, wird vorsichtig eingeschoben und mit Klebestreifen am Kopf fixiert ( s. Kasten). Hierdurch werden auch die Atemwege bei eventuell auftretenden Blutungen freigehalten, denn Kaninchen sind obligate Nasenatmer.

Für die Inzisiviextraktion bevorzugt der Autor die Rückenlagerung. Allerdings sollte bei anästhesierten Kaninchen eine längere Rückenlage vermieden werden, denn die Atmung könnte durch das Darmkonvolut behindert sein. Der Kopf des Patienten wird vom Rechtshänder mit der linken Hand fixiert und leicht gestreckt. Vor der Extraktion der Inzisivi sollten alle notwendigen Korrekturen an den Backenzähnen, z.B. das Einschleifen der Bisslage, erledigt werden. Zur sicheren Arbeit gehört ein sorgfältiges Abstützen mit Händen und Unterarmen auf dem Tisch, gute Beleuchtung und bei Bedarf eine Lupenbrille.

\section{Extraktion der Unterkieferinzisivi}

Es hat sich bewährt, mit den Unterkieferinzisivi zu beginnen. Überlange Schneidezähne werden mit einer Diamanttrennscheibe auf etwa $1 \mathrm{~cm}$ supragingivale Länge gekürzt. Nach vorsichtiger Reinigung und lokaler Desinfektion (z.B. mit 0,1\% Chlorhexidinlösung) werden die Lippen beiseite geschoben und mit einem 
feinen spitzen Skalpell wird die Gingiva allseitig um jeden Zahn gelöst. Die Stichinzisionen werden vorsichtig nach und nach entlang der Zahnkronen bis in die knöcherne Alveole vertieft. Die geringgradigen Blutungen können mit Tupfern gestillt werden. Nun werden die passenden Luxatoren vorsichtig allseitig abwechselnd eng am Zahn entlang in den Parodontalspalt eingeführt und sukzessiv bis in die Tiefe der Alveole vorgeschoben. Dabei ist die Biegung jedes Zahnes genau mit dem Instrument nachzuvollziehen. Zweckmäßigerweise beginnt man mit dem sichelförmigen langen Luxator an der lateralen Fläche eines Inzisivus, wodurch ein kleiner Spalt für die folgende Lateralbewegung geschaffen wird ( Abb.7). Danach geht man zwischen den beiden Inzisivi mit dem gleichen Luxator ein. Man muss eng mesial entlang der interdentalen Zahnfläche leicht nach lateral gerichtet immer am Zahn entlang in die Tiefe gehen. Keinesfalls darf hier durch einen falschen Eindringwinkel die Alveole verpasst werden, was eine Verletzung der Unterkiefersymphyse zur Folge hätte. Auch darf keinesfalls zwischen den beiden Inzisivi gehebelt werden, was ebenfalls die Symphyse sprengen könnte ( $\triangleright$ Abb. 8).

Eine Kontrolle der Eindringtiefe erfolgt durch Vergleich mit dem Röntgenbild; idealerweise wird zwischendurch eine Kontrollaufnahme angefertigt ( $\triangleright$ Abb.9).
Auf der labialen und lingualen Zahnfläche wird entsprechend mit dem schaufelförmigen Luxator gelöst. Dabei muss sehr vorsichtig und ohne Kraft vorgegangen werden, um die Alveole nicht zu sprengen oder subkutan bzw. subperiostal abzukommen.

Die Alveolarspalten werden auf diese Weise allseitig durch die passenden Luxatoren aufgeweitet und die parodontalen Fasern zerrissen. Dabei ist es wichtig, die Instrumente keinesfalls zu drehen, was ein Sprengen des Alveolarknochens oder Brechen des Zahnes zur Folge hätte. Vielmehr wird man sich durch wiegende Kippbewegungen vorsichtig schneidend in die Tiefe vorarbeiten. Die dabei ausgelösten feinen intraalveolären Blutungen tragen zum Lösen der Fasern bei. Deshalb belässt man die Instrumente jeweils einige Sekunden im Alveolarspalt stecken (Tipp: laut bis zehn zählen!), um sie dann wieder auf der gegenüberliegenden Seite einzuführen. Keinesfalls darf man dabei Kraft anwenden, denn der Kiefer kann gesprengt werden. Zwischen den beiden Inzisivi muss sorgfältig ohne Hebeln der leicht divergierende Zahnverlauf nachgearbeitet werden, sodass es nicht zur Sprengung der Unterkiefersymphyse kommt.

Sind die Inzisivi ausreichend tiefgelockert, lassen sie sich durch Vor- und Zurückschieben weiter mobilisieren und

\section{Praxistipp \\ Die luxierten Inzisivi werden aus- schließlich mit den Fingern gefasst, um das Frakturrisiko zu senken. Der Kiefer wird von Rechtshändern mit der linken Hand umgefasst und der zu ziehende Zahn wird zwischen Daumen und Zeigefinger der rechten Hand im Pinzettengriff gefasst. Mit einer Dreh- bewegung wird der Zahn heraus- gezogen; dabei wird der Daumen der rechten Hand als „Umlenkrolle“ benutzt ( Video '느).}

dann mit einfachen Fingerkräften aus der Alveole herausziehen. Keinesfalls sollten die Inzisivi mit einer Zange gefasst werden, da die dabei freigesetzten Kräfte zur Splitterung und Fraktur des Zahnes führen. Falls nötig, z.B. bei einem sehr kurzen Zahn, kann man mit einer feinen Pinzette oder einer parallel fassenden Arterienklemme den Griff verbessern.

\section{Extraktion der Oberkieferinzisivi}

Nach der Extraktion der unteren Schneidezähne erfolgt die Extraktion der oberen Inzisivi. Hierbei ist zu beachten, dass deren Radius wesentlich kleiner ist und ein entsprechendes Werkzeug in engem Bogen mit der Zahnkrümmung geführt werden muss. Das Risiko, durch divergierende Schnittführung (via falsa,

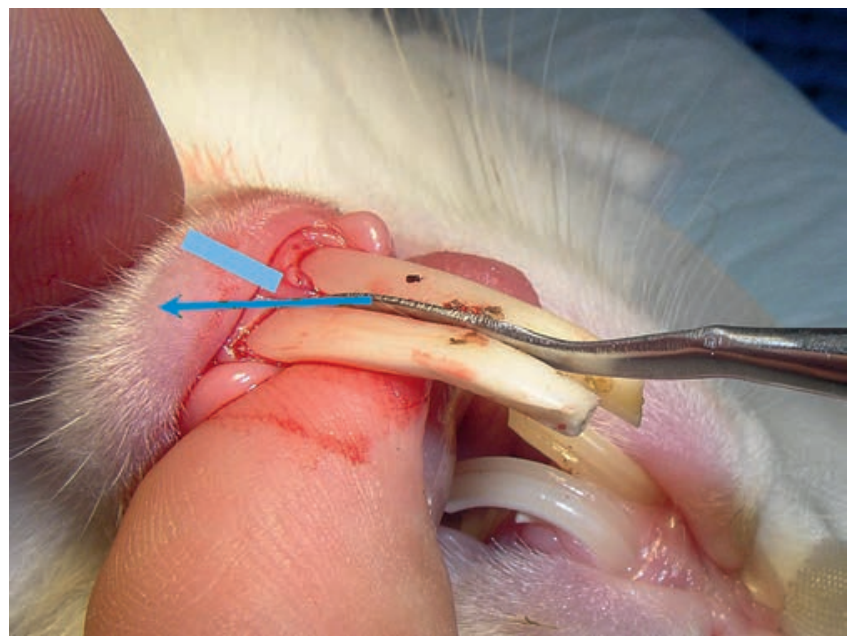

Abb. 8 Der Luxator wird interdental eingeführt und folgt streng der mesialen Zahnflanke (blauer Pfeil). Keinesfalls darf an dieser Stelle der Alveolarspalt verfehlt werden, weil sonst die Symphyse (hellblau) verletzt oder gesprengt werden kann. (C) S. Gabriel

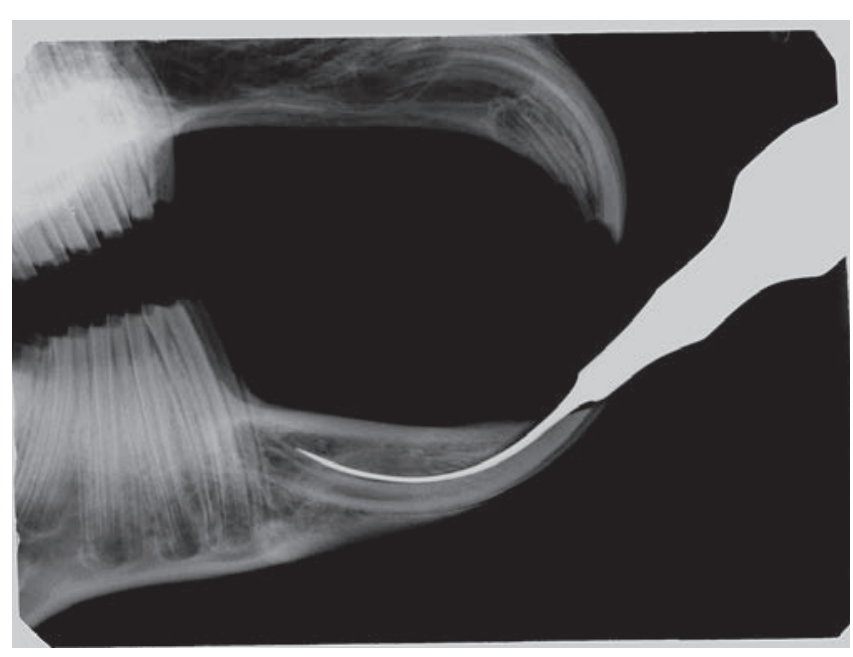

Abb. 9 Kontrollröntgenbild: Der flache schaufelförmige Luxator nach Fahrenkrug ist an der lingualen Zahnfläche bis auf die maximale Arbeitstiefe vorgeschoben worden und hat den Inzisivus etwas nach ventral gelockert. (C) S. Gabriel 


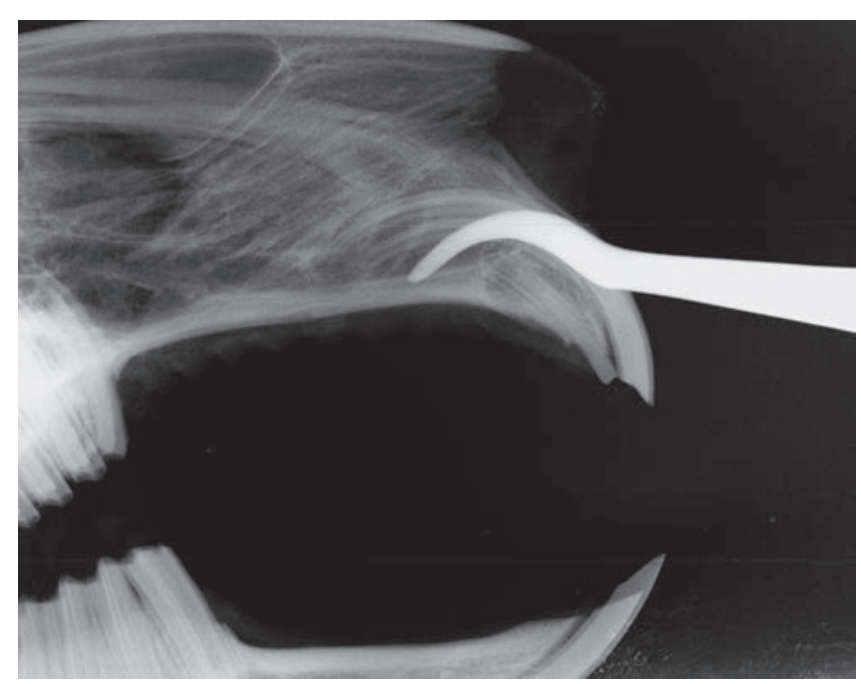

Abb. 10

Der Luxator wurde im falschen Radius vorgeschoben und hat den Parodontalspalt verfehlt (via falsa). (c) S. Gabriel

- Abb. 10) die Nasenhöhle zu verletzen, ist besonders groß.

Die Oberkieferinzisivi werden bei der Extraktion herausgedreht und nicht gezogen. Der Zug darf dabei nur axial im Bogen erfolgen, so wie man einen Krummsäbel aus der Scheide zieht.

Das geht besonders leicht bei guter Abstützung am Schädel, indem man den Zahn zwischen Daumen und Zeigefinger fasst und über den Daumen als Umlenkrolle dreht ( Video '

Die kleinen geraden Stiftzähne werden tief mit dem Skalpell losgeschnitten und gerade herausgezogen. Das geschieht zweckmäßigerweise vor der Extraktion der großen Inzisivi. Blutungen lassen sich durch Kompression stillen, die großen Inzisivi werden zur Blutstillung wieder für eine kurze Zeit in ihre Alveole

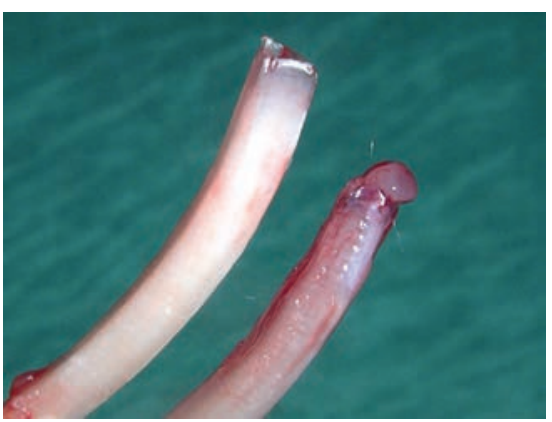

Abb. 11 Der untere extrahierte Inzisivus enthält seine Pulpa („Zahnsäckchen“). Der obere Inzisivus ist leer: Das bedeutet, dass die Pulpa in der Alveole verblieben ist. (C) S. Gabriel gesteckt, sodass sich ein natürlicher Thrombus bilden kann.

\section{Fehlerquellen und Komplikationen}

Die häufigsten Fehlerquellen sind Ungeduld und zu großer Kraftaufwand. Man muss sich einfach die nötige Zeit für die vollständige Luxation von allen Seiten nehmen. Besonders bei den stärker gekrümmten Oberkieferinzisivi ist es beim Herausziehen wichtig, genau im Verlauf der Zahnkrümmung und mit stetigem, aber nicht zu großem Kraftaufwand zu ziehen, um den Zahn nicht zu frakturieren.

Nach der Extraktion müssen alle Zähne an der Wurzel kontrolliert werden, ob die Pulpa (das sogenannte „Zahnsäckchen“) mit extrahiert wurde ( $\triangleright$ Abb.11). Falls der Zahn leer ist, ist die Pulpa in der Alveole verblieben, was ein Nachwachsen von Zahnsubstanz ermöglicht. In diesem Fall muss die Pulpa mit einer umgebogenen Kanüle kürettiert und herausgezogen werden. Gelingt das nicht, muss versucht werden, verbliebene Reste des germinativen Gewebes mechanisch (Curettage der Alveole oder wiederholtes Einstoßen des Zahnes) oder chemisch (Lotagen, Jod) zu zerstören. Eine Röntgenkontrollaufnahme liefert den Nachweis, dass alle Zahnhartsubstanzen entfernt werden konnten.

Auch bei vollständiger Extraktion gibt es ein gewisses Risiko, dass in der Tiefe der
Alveole germinatives Gewebe verblieben ist, das wieder Zahnhartsubstanz produzieren kann.

Auch eine negative Röntgenkontrolle nach der Extraktion ist keine Garantie dafür, dass keine Zahnsubstanz nachwachsen kann. Das passiert aber nur sehr selten. Man sollte den Tierhalter stets darauf hinweisen und zu einer Röntgenkontrolle nach einigen Wochen raten.

Wenn beim Extraktionsversuch ein Inzisivus abbricht, sollte man das Nachwachsen abwarten und dann eine erneute Extraktion durchführen. Bei schon luxierter Zahnwurzel kann man die Extraktion des Fragments mit einer Endo-Feile versuchen oder durch Trepanation eine offene Extraktion durchführen. In der Alveole steckengebliebene Wurzelfragmente nekrotisieren oft und werden durch ihre Fremdkörperwirkung und/oder eine bakterielle Infektion zur Ursache von Osteomyelitis.

Liegt bereits ein Zahnwurzelabszess vor ( $\triangleright$ Abb.2), muss die vereiterte Alveole nach der Zahnextraktion als Drainage der Abszesshöhle offen bleiben. Im Unterkiefer liegt häufig eine gemeinsame Abszedierung der benachbarten Wurzeln des Inzisivus und des 1. Backenzahns vor. Dieser sollte dann ebenfalls extrahiert und die Abszesshöhle vorzugsweise nach unten eröffnet und großzügig kürettiert werden. Über die Alveole des extrahierten Inzisivus lässt sich dann bis zum Ausgranulieren leicht mehrmals täglich eine antiseptische Spüllösung (z.B. PVP-Jod 2\%) einbringen.

\section{Post-OP und Langzeit- überwachung}

Postoperativ sollte an eine gute Analgesie für einige Tage gedacht werden.

Die Alveolen der extrahierten Inzisivi bleiben offen oder können zur Verhinderung von Futtereinspießung mit resorbierbarem Nahtmaterial (2-0 bis $5-0$ ) adaptiert werden. Eine saubere (nichtinfizierte) Alveole sollte nicht gespült oder mit Einlagen versorgt werden, um die natürliche Granulation aus dem Thrombus nicht zu stören. 


\section{Extraktion der Inzisivi}

1. Allgemeinnarkose und Analgesie

2. Schädelröntgen latero-lateral

3. Kürzen und Einschleifen der Backenzähne

4. Reinigung und Desinfektion des OP-Gebiets

5. Auswahl der passenden Luxatoren anhand der Röntgenaufnahme

6. Vorgeschlagene Reihenfolge: Unterkieferinzisivi, Stiftzähnchen, Oberkieferinzisivi

7. Inzision der Gingiva mit spitzem Skalpell allseitig bis in die Alveole

8. Erweiterung der Inzision mit passendem Luxator allseitig bis in die knöcherne Alveole

9. Abwechselndes Vorschieben des Luxators allseitig bis zum Wurzelbereich (jeweils 10 Sekunden stecken lassen, nicht hebeln oder rotieren)

10. GEDULD!

11. Fassen des gelockerten Zahns mit den Fingern, vorsichtige Extraktion im Bogen

12. Kontrolle an der Zahnwurzel, ob die Pulpa komplett entfernt wurde

13. Zurückstecken des extrahierten großen Inzisivus zur Blutstillung

14. Wenn die Pulpa nicht mit gezogen wurde: Kürettage, Spülung, Verödung

15. Wundnaht: Adaptation der Gingiva mit einem 2-0 bis 5-0 resorbierbaren Faden (bei vereiterter Alveole offen lassen)

16. Postoperative Röntgenkontrolle der Alveolen (Schädelröntgen latero-lateral)

17. Antibiose nur bei infizierter Alveole oder Abszessgeschehen

18. Postoperative Analgesie für 3-6 Tage

19. Baldiges Anfüttern nach Narkoseende und ggf. Handfütterung bis zur selbständigen Nahrungsaufnahme

20. Kontrolluntersuchung nach 1 Tag, 6 Tagen, 6 Wochen oder bei Komplikationen

21. Röntgenkontrolle nach 4-6 Wochen

22. Lebenslange regelmäßige Kontrolle der Backenzähne

Die infizierten Alveolen abszedierter Zähne sollten nach der Extraktion sorgfältig kürettiert, gespült und zur Drainage offen belassen werden. Eine perioperative Antibiose mit einem knochengängigen Antibiotikum zum Schutz vor septischer oder lokaler Keimausbreitung wird parenteral für einige Tage empfohlen. Von antibiotischen Einlagen ist

dringend abzuraten, da sie den Abfluss verstopfen, Biotope für Anaerobier schaffen und oral aufgenommen werden können.

Nach Abschluss der OP ist eine seitliche Röntgenaufnahme zur Kontrolle dringend anzuraten.

Tab. 1 Mögliche Komplikationen bei der Extraktion der Inzisivi bei Kaninchen.

\begin{tabular}{|c|c|c|}
\hline Mögliche Komplikationen & Ursachen & Lösungen \\
\hline Sprengung der Alveole & $\begin{array}{l}\text { - Falsche Kraftanwendung } \\
\text { Rotation oder Hebeln mit } \\
\text { dem Luxator }\end{array}$ & $\begin{array}{l}\text { - Röntgenkontrolle } \\
\text { - Analgesie }\end{array}$ \\
\hline $\begin{array}{l}\text { Nachwachstum von } \\
\text { Zahnsubstanz }\end{array}$ & $\begin{array}{l}\text { Verbliebenes germinatives } \\
\text { Gewebe }\end{array}$ & $\begin{array}{l}\text { - Röntgenkontrolle } \\
\text { - Nachwachsen abwarten } \\
\text { - Erneute Extraktion }\end{array}$ \\
\hline Fraktur des Inzisivus & $\begin{array}{l}\text { - Falsche Kraftanwendung } \\
\text { - Schmelzbildungsstörung } \\
\text { - Zahndeformation ( } \text { Abb. 12) }\end{array}$ & $\begin{array}{l}\text { - Nachwachsen abwarten } \\
\text { - Erneute Extraktion } \\
\text { - Röntgenkontrolle }\end{array}$ \\
\hline $\begin{array}{l}\text { Vereiterung der Alveole/ } \\
\text { Zahnwurzel }\end{array}$ & $\begin{array}{l}\text { - Primäres Abszessgeschehen } \\
\text { - Eitrige Pulpitis }\end{array}$ & $\begin{array}{l}\text { - Röntgenkontrolle } \\
\text { Radikale Chirurgie + } \\
\text { Antibiose }\end{array}$ \\
\hline
\end{tabular}

Operierte Patienten müssen so bald wie möglich wieder ans Fressen gebracht werden. Dazu können sie mit Breifutter und weichen Blattstückchen angefüttert werden. Die Tiere können nach der Entfernung der störenden funktionslosen Inzisivi meist spontan besser fressen. Jedoch müssen manche Patienten erst lernen, das Futter mit den Lippen aufzunehmen und mit den Backenzähnen zu zerteilen. Dazu eignen sich besonders Löwenzahnblätter, Möhrengrün oder mit dem Sparschäler hergestellte Möhrenstreifen.

Sobald der Patient selbständig Futter aufnimmt, kann er mit einer entsprechenden Einweisung der Tierbesitzer nach Hause gegeben werden. Das Tier sollte täglich gewogen und engmaschig tierärztlich kontrolliert werden. Manche Kaninchen haben ohne Inzisivi Probleme mit der Fellpflege und sollten vermehrt gebürstet werden.

Da das Überwachstum der Inzisivi in der Regel durch eine Malokklusion im Backenzahngebiss verursacht wurde, ist das Problem durch die Extraktion der Inzisivi allein noch nicht gelöst.

Der Besitzer muss darauf hingewiesen werden, dass regelmäßige tierärztliche Kontrollen (und ggf. Korrekturen) der Backenzähne notwendig sind, auch wenn der Patient zufriedenstellend frisst.

Bei einer routinemäßigen Kontrolluntersuchung nach 4-6 Wochen sollte man

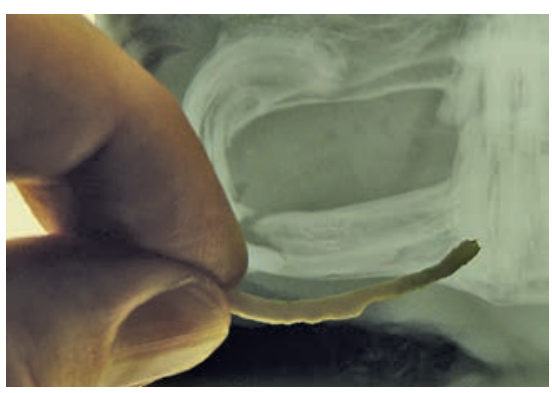

Abb. 12 Dieser Inzisivus hat durch periapikale Entzündungen phasenweise Wachstumsstörungen erlitten. Auf dieser rauen Schmelzoberfläche lässt sich der Luxator ausgesprochen schwer vorschieben. Beim Ausziehen frakturieren diese Zähne sehr leicht, wenn sie nicht zuvor allseitig perfekt gelöst wurden. (C) S. Gabriel 
eine seitliche Röntgenaufnahme anfertigen, um die leeren Alveolen auf Nachwachstum und die Backenzähne auf physiologische Abnutzung oder neuerliche Elongation zu kontrollieren. Auch klinisch unauffällige Patienten sollten langfristig mindestens 3-4 Mal jährlich tierärztlich kontrolliert werden.

Online mit zusätzlichem Video zu finden unter http://dx.doi.org/10.1055/s-0033-1361568

\section{'回}

Das Video zu diesem Beitrag finden Sie online unter: www.thieme-connect.de/ejournals bei Ihrer kleintier.konkret

\section{Literatur}

1 Böhmer E. Extraktion der Schneidezähne bei Kaninchen und Nagern - Indikation und Technik. Tierarztl Prax Ausg K 2003; 31: 309-320

2 Böhmer E, Crossley D. Objective interpretation of dental disease in rabbits, guinea pigs and chinchillas. Tierarztl Prax Ausg K 2009; 4: 250-260

3 Böhmer E. 2010. Zahnheilkunde bei Kaninchen und Nagern: Lehrbuch aund Atlas.1. Aufl. Stuttgart: Schattauer; 2010

4 Crossley D. Extraction of rabbit incisor teeth. ProcEurVetDentSoc Forum 1994

5 Gabriel S. Röntgendiagnostik bei Malokklusion des Kaninchens. Veterinärspiegel 2013; 1: 17-21

6 Gabriel S. Schmerztherapie beim Heimtier. Kompendium Kleintier 2010; 32-35
7 Harcourt-Brown F, Chitty J, Hrsg. BSAVA Manual of Rabbit Surgery, Dentistry and Imaging. 1. Aufl. Gloucester: John Wiley \& Sons; 2013

8 Hartmann M. Zahnextraktion hypsodonter Zähne bei Nagern und Hasenartigen. Veterinärspiegel 2007; 1: 22-27

9 Henke J. Analgesie und Anästhesie beim Kleinsäuger. Prakt Tierarzt 2010; 91: 4

10 Henke J, Müller D. Vollständig antagonisierbare Injektionsnarkose bei Heimtieren. Kleintiermedizin 2005; 5/6: 126-135. Im Internet: http://www.mueller-heinsberg.de/ Narkose_Nager_PDF.pdf

\section{Dr. Stefan Gabriel}

Tierärztliche Praxis für Kleintiere, Heimtiere, Zahnheilkunde

Le-Puy-Str. 13

59872 Meschede

dr.gabriel@t-online.de 